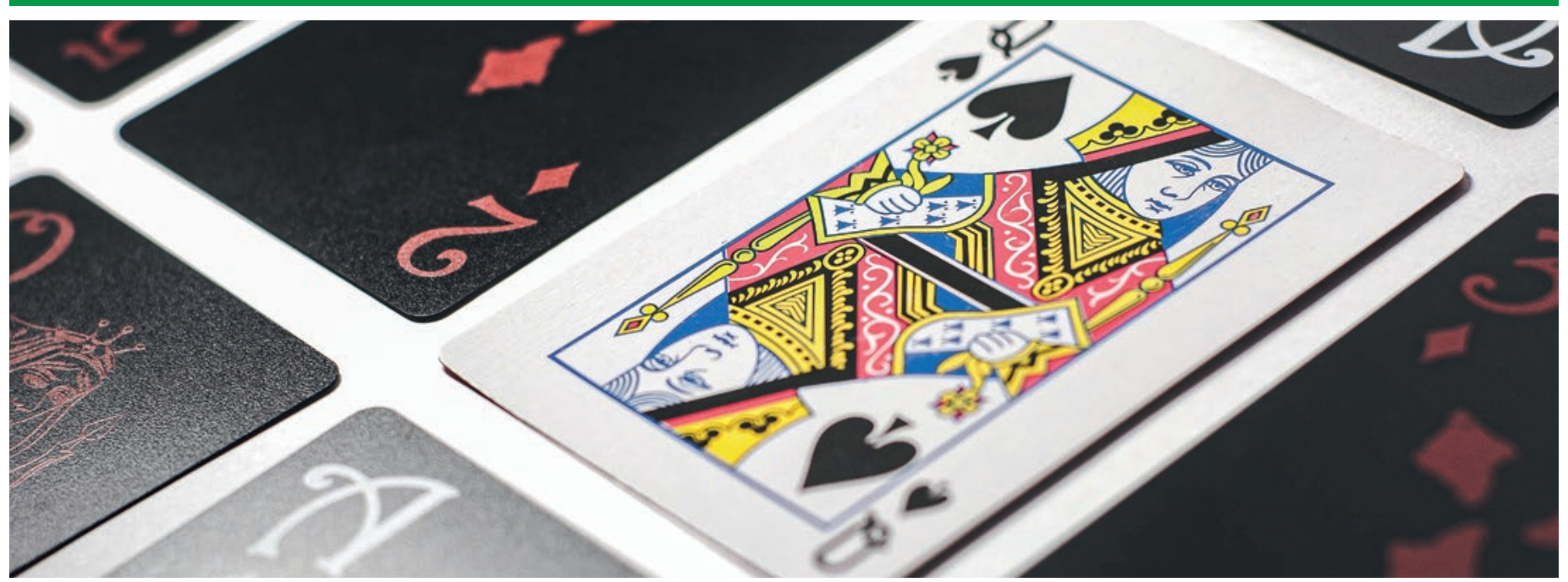

\title{
Die Zukunft des Führens in der Medizin
}

\section{Christina Venzin}

Dr. med., Leitende Ärztin Nephrologie, Spital Davos, Dozentin college M, Bern

Frauen stossen nach wie vor an die berüchtigte Glasdecke. Studien zeigen, dass normative Vorstellungen von Führung und «Weiblichkeit» eine Doppelbindung erzeugen, die Frauen benachteiligt. Dies zeigt sich in Bewerbungssituationen und bei der Förderung von Mitarbeitenden. Erst mit der Einsicht in die Voreingenommenheiten, die zu dieser Doppelbindung führen, können sich die Prozesse verbessern.

In Führungspositionen sind Frauen noch immer unterrepräsentiert - auch in der Medizin. Wie eine Studie im NEJM 2020 [1] zeigt, wurden zwischen 1979 und 1997 prozentual weniger Ärztinnen in Leitungspositionen befördert als ihre männlichen Kollegen - und die Lücke verschmälerte sich nicht in einer späteren Kohorte zwischen 1998 und 2013. Auch in der Schweiz sind Frauen prozentual weiterhin in Kaderpositionen unterrepräsentiert: In der Altersgruppe von 45 bis 59 sind Chefarztpositionen nur zu 13,4\% von Frauen besetzt.

Die herausfordernde Vereinbarkeit von Familie und Beruf ist nicht der alleinige Faktor für das Ungleichgewicht auf der Führungsebene.

Und das, obwohl der Anteil der weiblichen Ärzte in der Alterskategorie von 45 bis 54 Jahren $50 \%$ beträgt. In der Alterskategorie unter 40 Jahren übersteigt die Zahl der Medizinerinnen die der männlichen Kollegen; dennoch herrscht Ungleichgewicht: Nur 25\% der Leitungsfunktionen sind von Frauen besetzt [2]
Weshalb sollten Frauen seltener Leitende Ärztinnen und Chefärztinnen werden als ihre männlichen Kollegen? Die in der Schweiz noch immer herausfordernde Vereinbarkeit von Familie und Beruf ist ein nicht zu vernachlässigender Faktor, doch nicht der alleinige.

Unterschiedliche Erwartungen an männliche und weibliche Führungskräfte

Was wird in Führungspositionen erwartet, welche Führungsqualitäten sind gefordert? Eine ideale Führungskraft wird mit den Eigenschaften wie Entscheidungsfreude, Durchsetzungsfähigkeit, guter Vernetzung und Unabhängigkeit assoziiert - Eigenschaften, die man in der westlichen Kultur dem «idealen Mann» zuordnet. Frauen, die sich genau so verhalten, werden als kratzbürstig, schroff und unsympathisch wahrgenommen, denn sie sollen sich auch freundlich, fürsorglich und selbstlos zeigen. Dieses Dilemma kreiert eine sogenannte Doppelbindung, also eine widersprüchliche Erwartung, die unerfüllbar bleibt [3]. 
Eine berühmt gewordene Studie der Columbia University [4] konnte dies illustrieren: Studierende erhielten den Lebenslauf der sehr erfolgreichen Silicon-ValleyRisikokapitalgeberin Heidi Roizen. Die Hälfte der Studierenden erhielt denselben Lebenslauf mit geändertem Namen: Howard Roizen. Die Teilnehmenden, welche Fragen zu Howard beantworteten, bezeichneten ihn als beeindruckend und hätten gerne für ihn gearbeitet. Die Teilnehmenden, die den echten Lebenslauf von Heidi Roizen erhielten, fanden sie zwar kompetent, aber weniger sympathisch und wollten nicht für sie arbeiten.

Frauen müssen in Führungspositionen einen besonderen Balanceakt leisten, indem sie sich als starke Führungskraft einerseits und als weiblich genug andererseits darstellen, um sowohl als Führungskraft akzeptiert als auch sympathisch zu sein [5].

\section{Weshalb gibt es weniger Frauen in Führungspositionen?}

Positiv anzumerken ist, dass Stelleninserate in der Medizin nun (fast ausschliesslich) beide Geschlechter ansprechen; Kaderpositionen werden in Teilzeit angeboten, und Führungsqualitäten werden auf Ausgewogenheit bedacht formuliert (z.B. durchsetzungsfähig und kooperationsbereit). Doch: Bewerben sich gleich viele Frauen wie Männer auf eine Kaderposition? Das Ergebnis (die anteilsmässig geringe Vertretung von Frauen in der Führung) lässt zwei Dinge vermuten: Entweder Frauen bewerben sich seltener auf diese Positionen als Männer, oder sie stolpern im Laufe des Bewerbungsverfahrens über die gendered norms of leadership [6].

Zutreffend ist wohl beides. Aus Bereichen der Wirtschaft weiss man, dass Frauen sich tatsächlich seltener auf Kaderpositionen bewerben, da die Wahl in ein solches Gremium in der Regel mit psychologischen Sanktionen (Ablehnung, Sympathieentzug) einhergeht. "Psychologische Kosten» entstehen bei Frauen, die bereit sind, mit Männern in Konkurrenz zu treten [7].

\section{Rekrutierungsverfahren: Stolpersteine der Gendernormen in der Führungsetage}

Wenn sich Frau dann bewirbt, stellen Bewerbungsgespräche den ersten Stolperstein dar. In Männern sieht man das Potenzial, Frauen werden stärker nach ihren bisherigen Leistungen bewertet. Eine Studie untersuchte hierzu Bewerbungsverfahren und hielt fest: Um Berücksichtigung für eine Kaderposition zu erhalten, mussten die Leistungsnachweise von Frauen mindestens so gut sein wie die ihrer männlichen Kollegen, gleichzeitig mussten sie höheren Massstäben während des Auswahlverfahrens genügen, da das
Führungspotenzial bei Frauen häufig übersehen wurde [8].

So ist zwar die theoretische Möglichkeit zur Bewerbung auf eine Kaderarztstelle formal für Männer und Frauen in der Medizin gleich, doch weniger weibliche Bewerbungen und unterschiedliche Bewertungskriterien im Rahmen von Vorstellungs- und Auswahlgesprächen führen zu einem Ungleichgewicht.

Ein weiterer Stolperstein auf dem Weg zur weiblichen Kaderarztstelle sind Mitarbeitendengespräche. Eine Studie [9] untersuchte diese auf das Vorliegen von Mustern, die Mitarbeitendengespräche mit Männern von denen mit Frauen unterschieden: Persönlichkeitsmerkmale wurden deutlich häufiger bei Frauen er-

Rekrutierungsverfahren und Mitarbeitendengespräche sind Knackpunkte, die besondere Aufmerksamkeit verdienen.

wähnt als bei Männern, Frauen mussten das Erreichte nochmals beweisen (damit wiederholt sich das Muster aus den Bewerbungsgesprächen), und es galt die Annahme, dass Frauen mit Kindern weniger Karriereambitionen hätten als Männer mit Kindern (die sogenannte maternal wall). Zudem ermutigte man Männer häufiger, Visionen zu entwickeln und sich durchzusetzen, Frauen riet man eher zur Kooperation und dazu, den Fokus auf ein lieferbares Ergebnis zu richten.

Das Thema Selbstbewusstsein wird bei Frauen immer wieder bemängelt [10]. Warum wird Frauen häufiger unterstellt, sie seien nicht selbstbewusst? Man könnte auch umgekehrt davon ausgehen, dass Männer häufiger nach dem Motto fake it till you make it handeln und Frauen eine realistische Portion Selbstzweifel mitbringen [7].

\section{Die Stolpersteine beseitigen}

Muster und Ideale entstehen überall dort, wo es einfacher ist, in Kategorien zu denken, so auch bei den Gendernormen in der Führungsetage. Sie zu durchbrechen ist schwer, aber das Bewusstsein über ihren fragwürdigen Sinn in einer modernen Gesellschaft kann den Weg zu neuen Normen ebnen. Rekrutierungsverfahren und Mitarbeitendengespräche sind dabei Knackpunkte, die besondere Aufmerksamkeit verdienen. Wie können wir den Stolpersteinen konkret begegnen? Zumindest für die Mitarbeitendengespräche gibt es Evidenz [9]: Wenn man einerseits Bewertungsbögen so anpasst, dass mindestens drei konkrete Beispiele für die Bewertung einer Eigenschaft (z.B. Innovation, Führungsqualitäten) festgehalten werden müssen, und an- 
dererseits Mitarbeitende zum Hintergrund der Änderung schult, führt dies offenbar zu gleichberechtigterer Beförderung.

Und die übrigen Stolpersteine? Da sind Kreativität und Mut gefragt. Warum nicht einmal in Teilzeit auf eine Kaderposition bewerben - die Wahrscheinlichkeit, dass es nun immer mehr Pendants für Jobsharing geben wird, steigt stetig, schliesslich steigt der Wunsch nach Teilzeitarbeit bei beiden Geschlechtern. Auf Seite der Vorgesetzten wären Vorstellungsgespräche durch gemischte Teams denkbar. So sässe eine Bewerberin beiden Geschlechtern gegenüber, wodurch einerseits das Führungspotenzial einer Bewerberin seltener übersehen würde, gleichzeitig wäre das Signal auf Vorgesetztenseite klar: Wir bemühen uns um Perspektivenvielfalt.

\section{Welche Führungseigenschaften braucht die Medizin der Zukunft?}

Führung in der Medizin ist anspruchsvoll. Es gilt nicht nur, Mitarbeitende gut zu führen, auch Patientinnen und Patienten erwarten zunehmend andere Dinge von der Medizin. Eine Analyse von Patientenbefragungen [11] zeigt, dass Freundlichkeit, Respekt, Empathie, gute Koordination ihrer Behandlung und gute Kommunikation im Rahmen jeglicher Behandlungsform (ambulant und stationär) am stärksten wertgeschätzt werden. Dies gilt umso mehr, als sich Patientinnen und Patienten zunehmend durch einen medizinischen Dschungel bewegen müssen - noch nie zuvor gab es so viele unterschiedliche Behandlungsoptionen verschiedener Erkrankungen. Den Erwartungen nach Orientierung, Gesprächen und Augenhöhe mit klassischen, streng hierarchischen Führungseigenschaften begegnen zu wollen dürfte schwerfallen.

Der Medizinerberuf muss sich angesichts wachsender unterschiedlicher Erwartungen von Patientinnen und Patienten, Gesellschaft und Wirtschaft wandeln. Erfolgreiches und nachhaltiges Führen erfordert sowohl Fähigkeiten, die eher Frauen zugeschrieben werden, als auch klassische (eher mit Männern assoziierte)
Führungseigenschaften. Die Zusammenarbeit von Frauen und Männern ist auf allen Etagen erstrebenswert, da gemeinsam mehr Neues entstehen kann. Wenn wir das Führungspotenzial weiblicher Mitarbeitender verkennen, verschenken wir die Hälfte unseres gemeinsamen Potenzials. Und wir sollten unser volles Potenzial ausschöpfen, um das Gesundheitswesen in eine erfolgreiche Zukunft zu führen!

\section{Bildnachweis}

Esteban Lopez Unsplash

\section{Literatur}

1 Richter KP, Clark L, Wick JA, et al. Women Physicians and Promotion in Academic Medicine. N Engl J Med. 2020;383(22):2148-57. doi:10.1056/NEJMsa1916935

2 Poster FMH-Ärztestatistik 2020, https://www.fmh.ch/files/pdf25/ poster-fmh-aerztestatistik-2020.pdf

3 Ibarra H, Ely RJ, Kolb DM. Women Rising: The Unseen Barriers. Harvard Business Review. September 1st, 2013. Available at: https:// hbr.org/2013/09/women-rising-the-unseen-barriers (accessed: June 28, 2021).

4 Leadership psychology institute. Women \& the Leadership Labyrinth Howard vs Heidi. https://www.leadershippsychologyinstitute.com/women-the-leadership-labyrinth-howard-vs-heidi/

5 Koenig AM, Eagly AH, Mitchell AA, Ristikari T. Are leader stereotypes masculine? A meta-analysis of three research paradigms. Psychological Bulletin. 2011;137(4):616-42. doi:10.1037/a0023557

6 Zheng W, Kark R, Meister A. How Women Manage the Gendered Norms of Leadership. Harvard Business Review. Published online November 28, 2018. Accessed May 9, 2021. https://hbr.org/2018/11/ how-women-manage-the-gendered-norms-of-leadership

7 Berger J, Osterloh $\mathrm{M}$, Rost $\mathrm{K}$. Focal random selection closes the gender gap in competitiveness. Science Advances. 2020;6(47):eabb2142. doi:10.1126/sciadv.abb2142

8 Player A, Randsley de Moura G, Leite AC, Abrams D, Tresh F. Overlooked Leadership Potential: The Preference for Leadership Potential in Job Candidates Who Are Men vs. Women. Front Psychol. 2019;10. doi:10.3389/fpsyg.2019.00755

9 Williams JC, Loyd DL, Boginsky M, Armas-Edwards F. How One Company Worked to Root Out Bias from Performance Reviews. Harvard Business Review. Published online April 21, 2021. https:// hbr.org/2021/04/how-one-company-worked-to-root-out-biasfrom-performance-reviews

10 Doldor E, Wyatt M, Silvester J. Research: Men Get More Actionable Feedback Than Women. Harvard Business Review. Published online February 10, 2021. Accessed May 16, 2021. https://hbr. org/2021/02/research-men-get-more-actionable-feedback-thanwomen

11 Guney S, Childers Z, Lee TH. Understanding Unhappy Patients Makes Hospitals Better for Everyone. Harvard Business Review. Published online April 2, 2021. https://hbr.org/2021/04/understanding-unhappy-patients-makes-hospitals-better-for-everyone
Dr. med. Christina Venzin Spital Davos, Promenade 4 CH-7270 Davos Platz cvenzin[at]spitaldavos.ch

\section{Das Wichtigste in Kürze}

- Frauen müssen zusätzlich zu männlich attribuierten Führungsqualitäten wie Stärke auch weiblich konnotierte Qualitäten demonstrieren, um akzeptiert zu werden.

- Besondere Stolpersteine stellen Bewerbungsverfahren und die Förderung von Mitarbeitenden dar, da die Führungskompetenzen von Frauen oft übersehen werden.

- Diese Prozesse können durch die Verwendung von ausgewogenen Bewertungsbögen und durch gemischte Auswahlgremien offener gestaltet werden.

\section{L'essentiel en bref}

- Pour être acceptées, les femmes doivent faire preuve de qualités à connotation féminine en plus des qualités de leadership attribuées aux hommes, comme la force.

- Les processus de candidature et la promotion du personne constituent des obstacles fréquents, les compétences de leadership des femmes étant souvent négligées.

- $\quad$ Ces processus peuvent devenir plus accessibles par l'utilisation de formulaires d'évaluation équilibrés et par des panels de sélection mixtes. 